\title{
Curved polyhedra
}

\section{Hal Haggard*}

Centre de Physique Theorique

E-mail: haggardecpt.univ-mrs.fr

We describe how to reconstruct a constant curvature tetrahedron given four holonomies whose product is the identity. We construct a phase space describing the shapes of curved tetrahedra. We give a conjecture for the generalization of Minkowski's theorem to spaces of constant curvature. This establishes a new route to the description of four dimensional quantum gravity with a cosmological constant.

Frontiers of Fundamental Physics 14 - FFP14,

15-18 July 2014

Aix Marseille University (AMU) Saint-Charles Campus, Marseille

${ }^{*}$ Speaker. 